\title{
Bioesterified polysubstituted-cyclodextrin/surfactant nanoparticles obtained by multilevel self-assembly
}

\author{
L. Zerkoune ${ }^{1}$, A. Angelova ${ }^{1, *}$, L. Choisnard ${ }^{2}$, A. Gèze ${ }^{2}$, D. Wouessidjewe ${ }^{2}$ and S. Lesieur ${ }^{1}$ \\ ${ }^{1}$ CNRS UMR 8612 Institut Galien Paris-Sud, Univ Paris-Sud 11, 92296 Châtenay-Malabry, France \\ ${ }^{2}$ DPM, CNRS UMR 5063, ICMG FR 2607, UFR of Pharmacy, University of Grenoble, France \\ *e-mail: Angelina.Angelova@u-psud.fr
}

\begin{abstract}
The purpose of this work is to investigate the inclusion complexation between a novel amphiphilic biotransesterified cyclodextrin (CD), incorporated in nanostructured environment, and a model drug compound. A water-insoluble $\gamma$-cyclodextrin derivative $\left(\gamma-C D-C_{10}\right)$, polysubstituted with multiple $(n=7-8)$ decanoyl chains $\left(\mathrm{C}_{10}\right)$ on the secondary face, is produced by enzymatically-assisted esterification. The $\gamma-C D-C_{10}$ derivative is embedded in amphiphilic nanoenvironment created by self-assembly with the lipophilic dye Nile red (NR) and the non-ionic surfactant polysorbate 80 (P80). The inclusion complexation and the environmental effects upon the $\gamma$-CD$\mathrm{C}_{10} / \mathrm{NR} / \mathrm{P} 80$ nanoparticle (NP) formation, in a multilevel self-assembly approach, are investigated by means of steady-state fluorescence and Förster resonance energy transfer (FRET) techniques. Quasi-elastic light scattering (QELS) is used to control the NP size distribution during the sequential steps of the assembling process.
\end{abstract}

\section{Introduction}

Cyclodextrins (CDs) are recognized host materials capable of inclusion complex formation with guest waterinsoluble molecules, which are set in their hydrophobic nanocavities. This feature is being used to improve drug stability and bioavailability by increasing the drug permeability properties, solubility and/or dissolution through inclusion complexation, as well as to reduce the drug toxicity and side effects [1-9]. The formation of complexes between CDs and drug molecules via dynamic noncovalent interactions, in a host-guest model, has drawn considerable interest [1,9-12].

A recent methodology for grafting of alkyl chains on the secondary face of native CDs by enzymatic transesterification, using thermolysin as a biocatalyzer, has opened new possibilities for the design of drug nanocarriers with colloidal behaviour [8,9]. Amphiphilic $\mathrm{CD}$ derivatives with grafted decanoyl chains $\left(\mathrm{CD}-\mathrm{C}_{10}\right)$ may self-assemble into nanospheres with multilamellar inner organization or into reservoir-type particles, which can embed lipophilic drugs [5,7-9]. However, the unmodified $\mathrm{CD}-\mathrm{C}_{10}$ particles may readily aggregate depending on the surroundings. At variance, the modification of the $\mathrm{CD}-\mathrm{C}_{10}$ particles by nonionic surfactants, like polysorbate 80 (P80) (which involves poly(ethylene glycol) moieties in the polar head-group region) is anticipated to increase their steric stability, bioavailability, and cellular uptake. Towards this possibility, we here create mixed amphiphilic $\gamma$-CD$\mathrm{C}_{10} / \mathrm{P} 80$ assemblies and study the inclusion complexation mechanism with a fluorophore substance employed as a model drug.

The steps of the multilevel assembly approach leading to formation of polysubstituted-CDs/surfactant/ model-drug nanoparticles (NPs) are schematically presented in Fig. 1. The chemical structures of the investigated compounds are shown in Fig. 2. Nile red (NR) was chosen as a model drug (interacting with the $\mathrm{CD}$ cavity) because of its hydrophobic fluorophore and
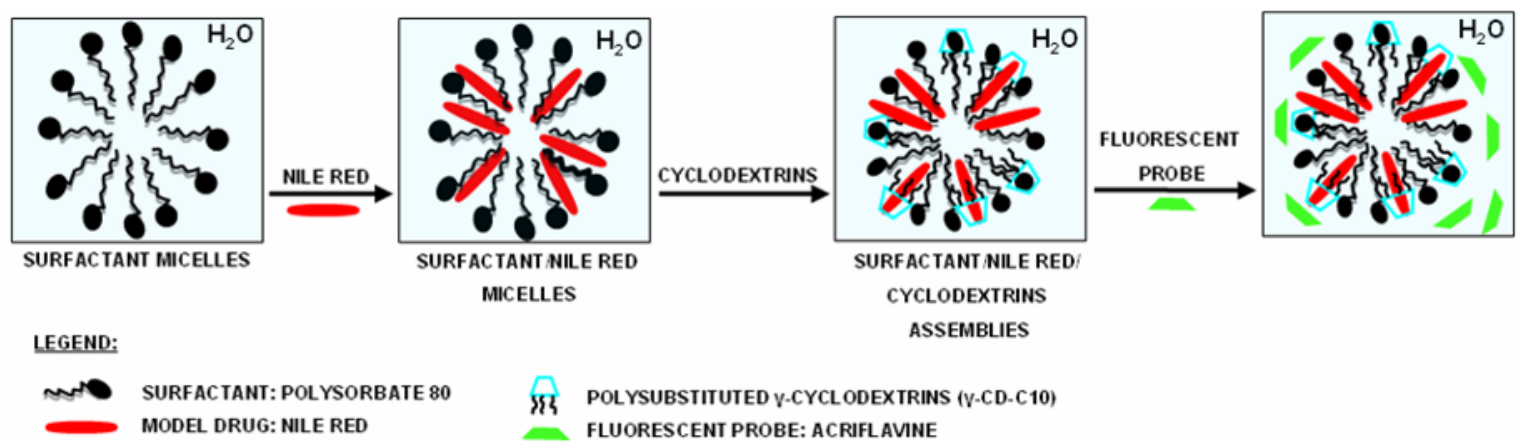

पदि POLYSUBSTITUTED V-CYCLODEXTRINS (V-CD-C10) MODEL DRUG: NILE RED FLUORESCENT PROBE: ACRIFL AVINE

Fig.1. Scheme of the multilevel self-assembly process at a low molar content of $\gamma-C D-C_{10}$. 


\subsection{Micelles preparation}

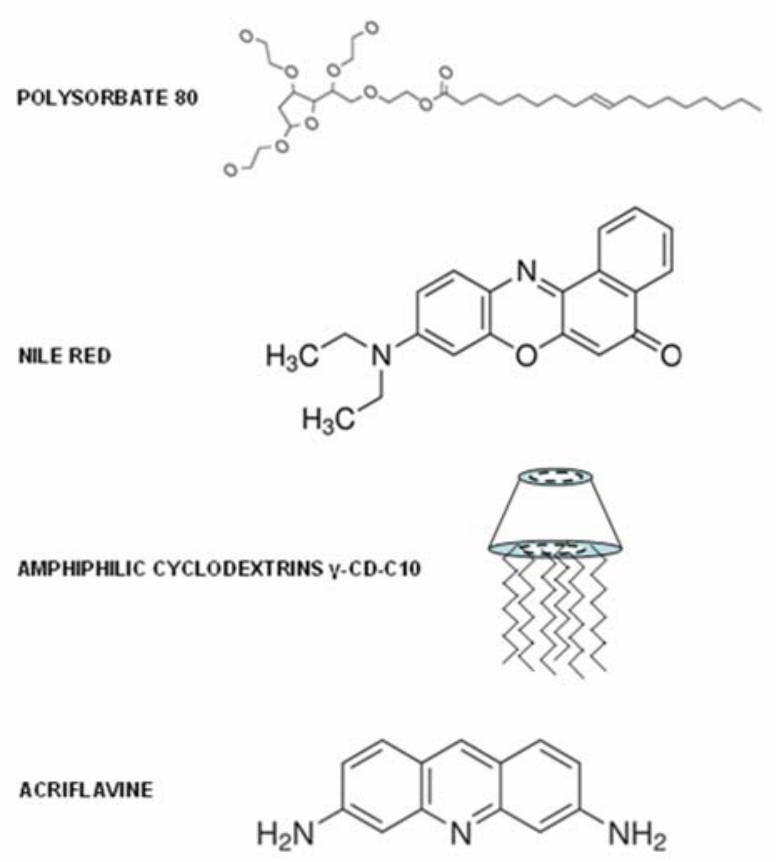

Fig.2. Structural formulae of the investigated molecules.

its stable fluorescence. NR has been previously studied as a polarity-sensitive fluorescence probe suitable for monitoring of structural transformations and intermolecular interactions upon micropolarity changes in the surroundings $[13,14]$.

The formation of an inclusion complex between the $\gamma-\mathrm{CD}-\mathrm{C}_{10}$ molecules and the guest fluorophore $\mathrm{NR}$ is studied here by fluorescence techniques. Fluorescence and UV-Visible spectroscopies have been appropriate methods for establishing the formation of inclusion complexes between CDs and fluorophores [15-17]. Here nanostructured environment of PEGylated micelles was prepared using the non-ionic surfactant P80 (Fig. 2). NR was added to the medium in order to form micelles incorporating the fluorophore (Fig. 1). Then, different amounts of cyclodextrins $\left(\gamma-C D-C_{10}\right)$ were added in order to study the complex formation between the lipophilic NR and the CD cavities in nanostructured surroundings. Förster resonance energy transfer (FRET) was investigated between the donor dye acriflavine (AC) (Fig. 2 ), included in the aqueous phase, and the acceptor NR dye incorporated in the surfactant micelles or in the $\gamma$ CD- $\mathrm{C}_{10}$-containing nanoassemblies.

\section{Experimental section}

\subsection{Materials}

Polysorbate 80 (P80), Nile red (NR), and acriflavine (AC) were purchased from Sigma-Aldrich. The amphiphilic $\gamma$-cyclodextrin derivative, $\gamma-C D-C_{10}$, was enzymatically synthesized as described by Choisnard et al. [9]. The average molecular weight, $\mathrm{M}_{\mathrm{n}}$, of the $\gamma-\mathrm{CD}$ $\mathrm{C}_{10}$ ester was $2505 \mathrm{~g} / \mathrm{mol}$ as determined by MALDI-TOF analysis.
$100 \mathrm{ml}$ of P80 micellar solution was prepared at concentration $10 \mathrm{mM}$ in $\mathrm{pH} 7.4$ phosphate buffer (solution A). $0.036 \mathrm{~g}$ of hydrophobic NR was added to 50 $\mathrm{ml}$ of solution A. This yielded $2.25 \mathrm{mM}$ dye concentration in P80 micellar medium (solution B).

\subsection{Preparation of $\mathrm{Y}-\mathrm{CD}-\mathrm{C}_{10} / \mathrm{P} 80$ mixed assemblies incorporating the guest NR}

NPs dispersions were prepared at different molar ratios of $\gamma$-CD- $\mathrm{C}_{10}$ with regard to P80. Briefly, $2.5 \mathrm{mg}, 5 \mathrm{mg}, 7.5$ $\mathrm{mg}$ or $10 \mathrm{mg}$ of $\gamma-\mathrm{CD}-\mathrm{C}_{10}$, corresponding to $0.9 \mathrm{~mol} \%$, $1.8 \mathrm{~mol} \%, 2.9 \mathrm{~mol} \%$, and $3.5 \mathrm{~mol} \%$ of cyclodextrins, were added to $14900 \mu \mathrm{l}$ of solution A (solution C). Then, $100 \mu \mathrm{l}$ of solution $\mathrm{B}$ were added to solution $\mathrm{C}$ in order to obtain $15 \mathrm{ml}$ of $\gamma-\mathrm{CD}-\mathrm{C}_{10} / \mathrm{P} 80$ mixed assemblies incorporating the fluorophore NR. The resulting concentration of NR was equal to $1.5 \times 10^{-5} \mathrm{M}$ in this 15 $\mathrm{ml}$ preparation. Vortexing and sonication was applied to ensure mixing of the components in all cases.

\subsection{Fluorescence spectra measurements}

Excitation and emission spectra of the two dyes were recorded at room temperature by means of a spectrofluorimeter SPEX Fluorolog (model $16810.22 \mathrm{~m}$ ) with an excitation wavelength of $\lambda \mathrm{ex}=455 \mathrm{~nm}$ for acriflavine (AC) and of $\lambda e=543 \mathrm{~nm}$ for Nile red (NR). The excitation and emission spectra were normalized in order to display the overlapping of bands between the fluorescence emission of $\mathrm{AC}$ and the absorption spectrum of NR. The excitation and the emission spectra of AC were obtained in phosphate buffer $(\mathrm{pH}$ 7.4), whereas those of NR were acquired in P80 micelles. The AC and NR concentrations were $3.3 \times 10^{-8} \mathrm{M}$ and $5 \times 10^{-6} \mathrm{M}$, respectively.

\subsection{FRET measurements}

Steady-state fluorescence spectra measurements were performed with the $\gamma-\mathrm{CD}-\mathrm{C}_{10} / \mathrm{NR} / \mathrm{P} 80$ nanoassemblies in the presence of $\mathrm{AC}$ (donor dye) in the aqueous phase using the same equipment. Briefly, $1 \mathrm{ml}$ solution of NRlabelled $\gamma-\mathrm{CD}-\mathrm{C}_{10} / \mathrm{P} 80$ mixed micelles, incorporating amphiphilic CDs with a varying molar fraction, was dropped down in a spectroscopy cell containing $1980 \mu \mathrm{l}$ of phosphate buffer (pH 7.4). For the FRET study, $20 \mu 1$ of acriflavine stock solution $\left(\mathrm{C}_{\text {stock }}=0.05 \mathrm{mM}\right)$ were added to the spectroscopy cell. The resulting AC and NR concentrations, in the spectroscopy cell, were of $3.3 .10^{-8}$ $\mathrm{M}$ and $5.10^{-6} \mathrm{M}$, respectively. FRET was recorded for the $\mathrm{AC} / \mathrm{NR}$ donor/acceptor pair at room temperature. The excitation wavelength was set at $480 \mathrm{~nm}$ and the emission spectra were collected from 500 to $800 \mathrm{~nm}$.

\subsection{Size measurements}

The samples studied by fluorescence spectroscopy were analysed also by quasi-elastic light scattering (QELS). The Zetasizer Nano ZS-90 system was a MALVERN instrument (light source He-Ne laser $633 \mathrm{~nm}$, maximum 
power $5 \mathrm{~mW}$ ). Every sample was measured three times. The scattering angle was fixed at $90^{\circ}$ and the temperature was $25^{\circ} \mathrm{C}$. The concentrations of the samples were the same as for the fluorescence experiments for either the initial P80/NR micelles or the $\gamma-\mathrm{CD}-\mathrm{C}_{10} / \mathrm{NR} / \mathrm{P} 80$ mixed nanoassemblies.

\section{Results and discussion}

\subsection{Fluorescence spectra of the studied dyes}

Figure 3 characterizes the AC/NR FRET pair. It shows that the emission spectrum of the donor dye, acriflavine (AC), overlaps with the excitation spectrum of the acceptor dye, Nile red (NR). The established good spectral overlap implies that it should be possible to observe a FRET event from acriflavine to Nile red when the two fluorophores come in a sufficient proximity in the investigated nanostructured system. In fact, FRET occurs when the electronic excitation energy of a donor chromophore is transferred to an acceptor molecule, located nearby, through dipole-dipole interaction occurring in the donor-acceptor pair.
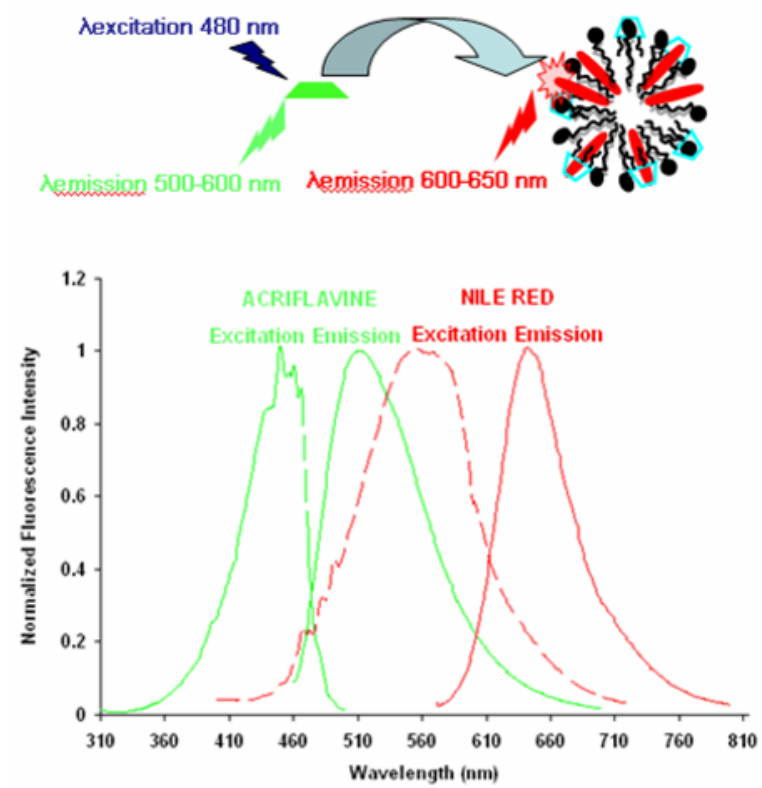

Fig.3. Overlapping between the fluorescence emission of the donor dye (acriflavine) in a phosphate buffer and the absorption spectrum of the acceptor molecule (Nile red) in P80 micelles. The excitation wavelengths were $455 \mathrm{~nm}$ and $543 \mathrm{~nm}$ for acriflavine and Nile red, respectively. The emission spectra were recorded in the range $460-700 \mathrm{~nm}$ and $570-800 \mathrm{~nm}$ for the two dyes, respectively.

\subsection{Mechanism of inclusion complexation in NPs}

In the investigated here colloidal nanoassemblies, the components of the FRET pair are located in different environments. $\mathrm{AC}$ is water soluble, while $\mathrm{NR}$ is incorporated in the mixed assemblies and may be anchored in the nanocavities of the $\gamma-\mathrm{CD}-\mathrm{C}_{10}$. The donor dye AC surrounds the P80/NR micelles as well as the $\gamma$ CD-C $10 / \mathrm{NR} / \mathrm{P} 80$ NPs (Fig. 1, right). A greater proximity of the two dyes, and hence more efficient FRET, would be achieved if the acceptor (NR) is located at the polar/apolar interfaces of the $\gamma-\mathrm{CD}-\mathrm{C}_{10} / \mathrm{NR} / \mathrm{P} 80$ nano assemblies rather than solubilized deeply in the core of the micelles or the NPs formed at increasing CDs content.

Figure 4 shows that the donor dye AC is strongly fluorescent with a maximum emission at $510 \mathrm{~nm}$. The fluorescence intensity of AC decreases sharply upon the addition of NR-labelled micelles (spectrum 2). The NR fluorescence emission was observed at $630 \mathrm{~nm}$.
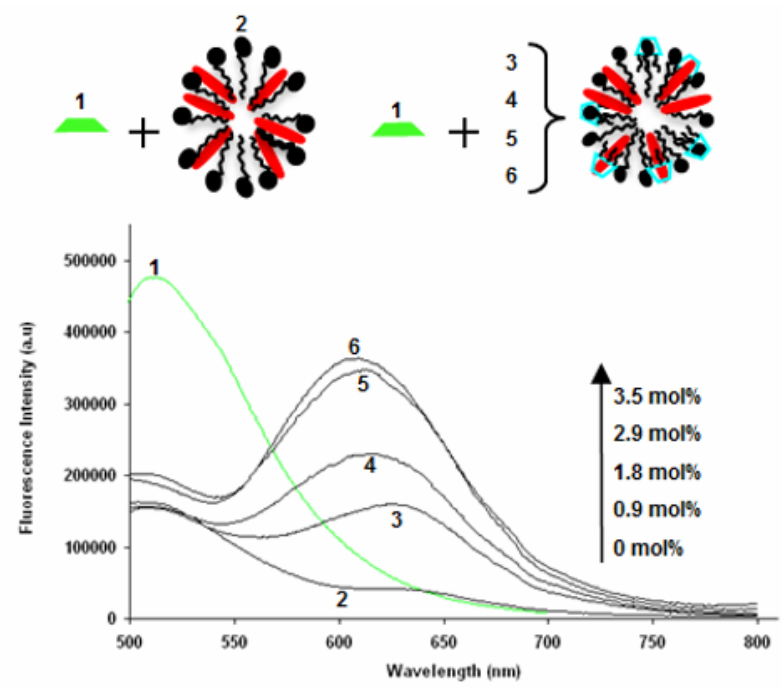

Fig.4. Fluorescence emission spectra of (1) acriflavine in phosphate buffer ( $\mathrm{pH} 7.4$ ) and in (2) Nile red/P80 micelles. The $\mathrm{AC}$ concentration is $3.3 .10^{-8} \mathrm{M}$ and the NR concentration is $5.10^{-6} \mathrm{M}$. The fluorescence emission maximum of $\mathrm{NR}$ is enhanced at increasing concentrations of amphiphilic CDs. The $\gamma$-CD- $\mathrm{C}_{10}$ molar fraction is varied as (2) $0 \mathrm{~mol} \%$, (3) $0.9 \mathrm{~mol} \%$, (4) $1.8 \mathrm{~mol} \%$, (5) $2.9 \mathrm{~mol} \%$, (6) $3.5 \mathrm{~mol} \%$ with respect to P80 (see Inset). The excitation wavelength for AC is $480 \mathrm{~nm}$.

Spectra 3 to 6 show the progressive increase in the emission maximum intensity of NR upon augmenting the molar fraction of $\gamma-\mathrm{CD}-\mathrm{C}_{10}$ molecules in the NPs. The concentration of NR is constant in all cases. Hence, if NR has remained located at the same distance in the mixed structures (3-6) from the AC acceptor, the spectral intensities would be always equal to that in spectrum 2 . However, one observes an increase in the fluorescence emission of NR and a blue shift of its fluorescence peak maximum at increasing molar fractions of amphiphilic $\gamma$ $\mathrm{CD}-\mathrm{C}_{10}$ molecules, which present cavities for inclusion complexation. The fluorescence of NR is essentially stronger in the obtained NP assemblies with $\gamma-\mathrm{CD}-\mathrm{C}_{10}$ as compared to that in P80 micelles. Evidently, inclusion complexes are formed between $\gamma-\mathrm{CD}-\mathrm{C}_{10}$ and $\mathrm{NR}$ in nanoconfined geometries.

The increase in the fluorescence emission of NR and the blue shift of the NR peak maximum are more significant at higher molar fractions of $\gamma-C D-C_{10}$. This indicates an increased hydrophobicity of the NR surroundings and changes in the micropolarity around the NR fluorophore in the presence of CDs. Altogether, these results confirm that there is an inclusion complex formation between the hydrophobic nanocavities of the CDs and the guest model drug NR. 

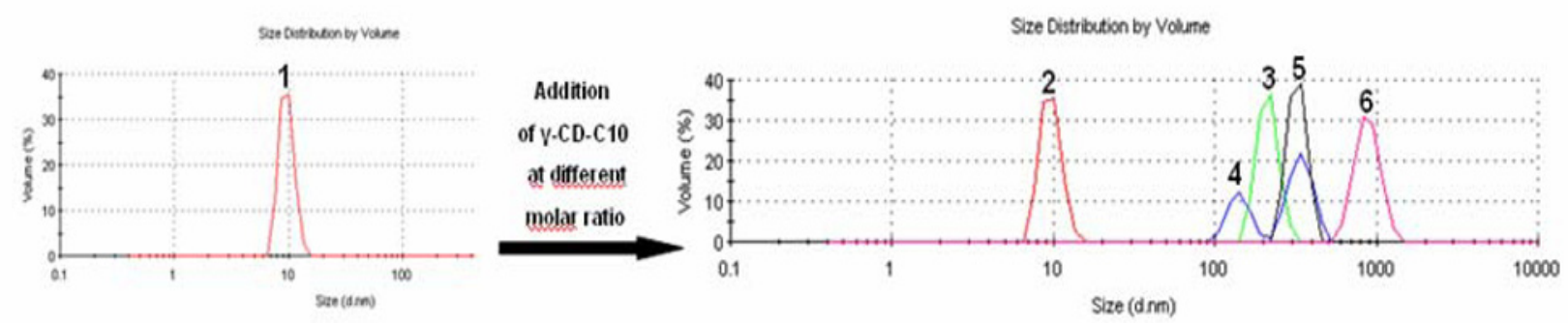

Fig.5. Particle size distributions of polysorbate $80 /$ Nile red micelles ( 1 , red curve) and of polysorbate $80 / \mathrm{Nile}$ red $/ \gamma$-CD-C 10 mixed assemblies with $0 \mathrm{~mol} \% \mathrm{CD}$ (2, red curve), $0.9 \mathrm{~mol} \% \mathrm{CD}$ (3, green curve), $1.8 \mathrm{~mol} \% \mathrm{CD}$ (4, blue curve), $2.9 \mathrm{~mol} \% \mathrm{CD}$ (5, black curve) and $3.5 \mathrm{~mol} \% \mathrm{CD}(6$, pink curve).

At a low molar fraction of $\gamma$-CD-C $10(<1 \mathrm{~mol} \%)$, the NR/CDs inclusion complexation most likely occurs at the polar/apolar interfaces of mixed micelles. Under these conditions, the proximity of NR to $\mathrm{AC}$ (which is situated in the aqueous phase) is highest and the attenuation of the AC fluorescence peak is maximal. The effects observed at higher molar fractions of $\mathrm{CDs}$ require a consideration of the NP sizes obtained upon $\gamma-\mathrm{CD}-\mathrm{C}_{10} / \mathrm{NR} / \mathrm{P} 80$ selfassembly (Fig. 5).

\subsection{Nanoparticle sizes}

Quasi-elastic light scattering (QELS) was employed for determination of the particles sizes and distributions upon formation of mixed nanoassemblies with amphiphilic $\gamma$ $\mathrm{CD}-\mathrm{C}_{10}$. Figure 5 shows that there is an increase in the size of the P80/NR assemblies upon addition of CDs. This result confirms that there is an interaction between the amphiphilic CDs and the P80/NR micelles. It also suggests a structural change in the NP organization at increasing $\mathrm{CDs}$ content. The formation of $\gamma-\mathrm{CD}$ $\mathrm{C}_{10} / \mathrm{NR} / \mathrm{P} 80$ assemblies leads to NP sizes in the range from $140 \mathrm{~nm}$ to $825 \mathrm{~nm}$. Indeed, NR embedded in large NPs, different from micelles, cannot only be located at the polar/apolar interfaces. Its inclusion in the NPs interior will increase the distance to the $\mathrm{AC}$ fluorophore. Hence, the reduction of the AC fluorescence intensity should be smaller for bigger NPs.

\section{Conclusions}

The performed study demonstrated the inclusion complexation between novel biotransesterified amphiphilic cyclodextrins and a model drug (NR) in nanostructured environment created by multilevel selfassembly. A new FRET pair (AC donor/NR acceptor) was proposed for probing the location of the guest NR in P80 micelles or in the CDs nanocavities in NPs. Both the QELS and the fluorescence measurements were sensitive to the NP organization at varying $\gamma-\mathrm{CD}-\mathrm{C}_{10}$ content. The obtained results provided evidence for the occurrence of host-guest type of interactions in a nanostructured medium. The structural organization of the new NPs requires a separate investigation.

\section{References}

1. M.E. Davis, M.E. Brewster, Nat. Rev. Drug Discov. 12, 1023-1035 (2004).

2. C.M. Fernandes, V.M. Teresa, F.J. Veiga, Eur. J. Pharm. Sci. 15, 79-88 (2002).

3. S.W. Jun, M.S. Kim, H.J. Park, S. Lee, J.S. Woo, S.J. Hwang, Eur. J. Pharm. Biopharm. 66, 413-421 (2007).

4. S.M. Badr-Eldin, S.A. Elkheshen, M.M. Ghorab, Eur. J. Pharm. Biopharm. 70, 819-827 (2008).

5. S. Lesieur, D. Charon, P. Lesieur, C. RingardLefebvre, V. Muguet, D. Duchêne, D.Wouessidjewe, Chem. Phys. Lipids. 106, 127-144 (2000).

6. A. Angelova, C. Fajolles, C. Hocquelet, F. DjedaïniPilard, S. Lesieur, V. Bonnet, B. Perly, G. Le Bas, L. Mauclaire, J. Colloid Interface Sci. 322, 304-314 (2008).

7. L. Choisnard, A. Gèze, J.L. Putaux, Y.S. Wong, D. Wouessidjewe D. Biomacromolecules 7, 515-520 (2006).

8. L. Choisnard, A. Gèse, C. Vanhaverbeke, J.B.G. Yamégo, J.L. Putaux, B. Brasme, L. Julien, S. Boulanger, C. Elfakir, D. Wouessidjewe, Biomacromolecules, 12, 3031-3038 (2011).

9. J.B.G. Yaméogo, A. Gèze, L. Choisnard, J.L. Putaux, A. Gansané, S.B. Sirima, R. Semdé, D. Wouessidjewe, Eur. J. Pharm. Biopharm. 80, 508517 (2012).

10. R. Singh, N. Bharti, J. Madan, S.N. Hiremath, J. Pharm. Sci. Tech. 2, 171-183 (2010).

11. A. Angelova, C. Ringard-Lefebvre, A. Baszkin, J. Colloid Interface Sci. 212, 275-279 (1999).

12. A. Angelova, C. Ringard-Lefebvre, A. Baszkin, J. Colloid Interface Sci. 212, 280-285 (1999).

13. M. Valero, I. Grillo, C.A. Dreiss, J. Phys. Chem. B 116, 1273-1281 (2011).

14. B.D. Wagner, P.G. Boland, J. Lagona, L. Issacs, J. Phys. Chem. B 109, 7686-7691 (2005).

15. S. Rakshit, S. Vasudevan, J. Am. Chem. Soc. 7, $1473-1479(2008)$.

16. A. Okamoto, K. Tainaka, Y. Fujiwara, JOC article.71, 3592-3598 (2006).

17. E.Iglesias, Photochem.Photobiol.Sci. 10, 531 (2011). 